\title{
DNA Methylation and All-Cause Mortality in Middle-Aged and Elderly Danish Twins
}

\author{
Anne Marie Svane ${ }^{1}$, Mette Soerensen ${ }^{1}$, Jesper Lund ${ }^{1}$ (D), Qihua Tan ${ }^{1,2}$, Juulia Jylhävä ${ }^{3}$ (D), \\ Yunzhang Wang ${ }^{3}$, Nancy L. Pedersen ${ }^{3}$, Sara Hägg ${ }^{3}$ (D), Birgit Debrabant ${ }^{1}$, Ian J. Deary ${ }^{4,5}$, \\ Kaare Christensen ${ }^{1,2}$, Lene Christiansen ${ }^{1}$ and Jacob B. Hjelmborg ${ }^{1, *}$ iD \\ 1 Epidemiology and Biostatistics, Department of Public Health, University of Southern Denmark, \\ 5000 Odense, Denmark; amsvane@health.sdu.dk (A.M.S.); msoerensen@health.sdu.dk (M.S.); \\ jlund@health.sdu.dk (J.L.); qtan@health.sdu.dk (Q.T.); bdebrabant@health.sdu.dk (B.D.); \\ KChristensen@health.sdu.dk (K.C.); lchristiansen@health.sdu.dk (L.C.) \\ 2 Unit of Human Genetics, Department of Clinical Research, University of Southern Denmark, 5000 Odense, \\ Denmark \\ 3 Department of Medical Epidemiology and Biostatistics, Karolinska Institutet, SE-171 77 Stockholm, Sweden; \\ juulia.jylhava@ki.se (J.J.); yunzhang.wang@ki.se (Y.W.); nancy.pedersen@ki.se (N.L.P.); \\ Sara.Hagg@ki.se (S.H.) \\ 4 Department of Psychology, University of Edinburgh, Edinburgh EH8 9JZ, UK; i.deary@ed.ac.uk \\ 5 Center for Cognitive Aging and Cognitive Epidemiology, University of Edinburgh, lEdinburgh EH8 9JZ, UK \\ * Correspondence: JHjelmborg@health.sdu.dk; Tel.: +45-65503075
}

Received: 29 September 2017; Accepted: 25 January 2018; Published: 8 February 2018

\begin{abstract}
Several studies have linked DNA methylation at individual CpG sites to aging and various diseases. Recent studies have also identified single CpGs whose methylation levels are associated with all-cause mortality. In this study, we perform an epigenome-wide study of the association between CpG methylation and mortality in a population of 435 monozygotic twin pairs from three Danish twin studies. The participants were aged 55-90 at the time of blood sampling and were followed for up to 20 years. We validated our results by comparison with results from a British and a Swedish cohort, as well as results from the literature. We identified $2806 \mathrm{CpG}$ sites associated with mortality (false discovery rate (FDR) < 0.05), of which 24 had an association $p$-value below $10^{-7}$. This was confirmed by intra-pair comparison controlling for confounding effects. Eight of the 24 top sites could be validated in independent datasets or confirmed by previous studies. For all these eight sites, hypomethylation was associated with poor survival prognosis, and seven showed monozygotic correlations above $35 \%$, indicating a potential moderate to strong heritability, but leaving room for substantial shared or unique environmental effects. We also set up a predictor for mortality using least absolute shrinkage and selection operator (LASSO) regression. The predictor showed good performance on the Danish data under cross-validation, but did not perform very well in independent samples.
\end{abstract}

Keywords: DNA methylation; mortality; EWAS; survival analysis; survival prediction; twin study; heritability

\section{Introduction}

Epigenetics have become very important in our understanding of aging processes. DNA methylation is an epigenetic mechanism that affects gene expression, and changes in the methylation pattern have been linked to various diseases [1-4]. DNA methylation is partly inherited, and partly influenced by environmental and random factors, as well as natural aging.

The degree of DNA methylation at certain CpG sites has been shown to change consistently with age in various organs and in the blood. This has led to the establishment of epigenetic "clocks" 
that predict chronological age from methylation patterns $[5,6]$. In fact, several collections of CpG sites for which the methylation level associates very well with age have been identified, suggesting that fundamental dynamical processes are reflected in, or at least associated with, such subsets of sites. Although the functioning of these sites is largely unknown, it is found that they also can be associated with all-cause mortality, i.e., age at death. Interestingly, previous studies have found that the deviation between methylation age as defined in $[5,6]$ and chronological age is associated with all-cause mortality $[7,8]$.

Several recent epigenome-wide association studies (EWAS) have identified individual CpG sites related to all-cause mortality. In [9], $58 \mathrm{CpG}$ sites linked to mortality are reported, and 10 of these are used to construct a mortality risk score that can be validated in independent data. CpG sites connected with mortality in very old people (above 90) are discovered in [10], and in [11] single CpGs are associated with mortality and compared to age-related CpGs. In all three studies, the CpG sites most strongly associated with mortality are different from those typically associated with age.

In this study, we perform EWAS analysis of DNA methylation in blood and survival data for 870 monozygotic (MZ) twins from the Danish Twin Register. Exploiting the fact that twins are naturally matched with respect to genes and early childhood environment, confounding of the association between site-specific methylation and survival time may be reduced by adjusting for twin pairing. To model within-pair dependence, we use a Cox frailty model to assess the influence of individual sites on time to death. Sites selected under false discovery rate criteria are compared to findings in the literature. Furthermore, a mortality predictor is constructed and validated in independent datasets. Random selection of twin pairs from those available allows us to use the twin design to obtain an upper limit for the genetic influence on methylation of mortality-associated CpG sites.

\section{Materials and Methods}

\subsection{Study Population}

The study comprises data on 435 pairs of monozygotic twins from three datasets collected by the Danish Twin Register: a study of extremely birth weight-discordant twins (BWD) [12], the Longitudinal Study of Aging Danish Twins (LSADT) [13], and the Middle Aged Danish Twins Study (MADT) [14]. Blood samples were taken over the periods 1996-1997 for LSADT and 2008-2011 for MADT and BWD. Dates of births and deaths were obtained from the Danish Civil Registration System in 2016. By then, 258 deaths had occurred. Table 1 shows the basic characteristics of the study population.

Table 1. Characteristics of the sample population. Cardiovascular diseases include apoplexy and age-related cardiovascular weakness.

\begin{tabular}{lcccc}
\hline Dataset & BWD & LSADT & MADT & Total \\
\hline$N$ & 150 & 238 & 482 & 870 \\
Women, $N(\%)$ & $74(49 \%)$ & $156(76 \%)$ & $218(46 \%)$ & 448 \\
Deaths, $N(\%)$ & $11(7.3 \%)$ & $213(89 \%)$ & $34(7.0 \%)$ & 258 \\
Age at blood sample & $57-74$ & $73-90$ & $55-79$ & $55-90$ \\
Year of blood sample & $2009-2010$ & $1996-1997$ & $2008-2011$ & \\
Follow-up year & 2016 & 2016 & 2016 & \\
\hline Cause of death: & & & & \\
$\quad$ Cancer & 1 & 35 & 4 & 40 \\
$\quad$ Cardiovascular disease & 0 & 72 & 2 & 74 \\
$\quad$ Respiratory causes & 1 & 18 & 1 & 20 \\
\hline
\end{tabular}

BWT: a study of birth weight-discordant twins; LSADT: Longitudinal Study of Aging Danish Twins; MADT: Middle Aged Danish Twins Study. 


\subsection{Methylation Data}

DNA was isolated from buffy coats using the salt precipitation method, and bisulfite conversion of $500 \mathrm{ng}$ genomic DNA was performed using the EZ Methylation Gold Kit (Zymo Research, Orange County, CA, USA). The methylation degree at 485,512 CpG sites was analyzed using the Infinium 450K HumanMethylation BeadChip (Illumina, San Diego, CA, USA) following the manufacturer's instructions at either the Leiden University Medical Center or GenomeScan B.V., Leiden, the Netherlands. BeadChip images were scanned using the iScan system. Twin pairs were analyzed on the same array. The three datasets were analyzed on different occasions.

Data preprocessing and quality control was performed in line with [15] using the R-package MethylAid. Samples not meeting the quality requirements were excluded. Probes with detection $p$-value $>0.01$, no signal, or bead count $<3$ were treated as missing. CpG sites with more than $5 \%$ missing values, probes targeting sex chromosomes, and cross-reactive probes were excluded from the analysis. Polymorphic sites were kept in the analysis, but polymorphic probes with allele frequency of least $1 \%$ in the European population [16] are marked in the results tables below. Data was normalized using functional normalization [17] with four principal components. After preprocessing, a total of 441,160 CpG sites remained for further analysis.

Blood cell type composition was measured for most MADT and BWD participants and this was used for imputing the proportion of basophils, eosinophils, monocytes, neutrophils, and lymphocytes in the remaining individuals (see [18] for details). The methylation beta values were corrected for batch effects and cell types using a linear mixed model with sex, age, dataset, and cell type proportions as fixed effects and with sample plate and plate position as random effects. Residuals were used in all subsequent analyses.

\subsection{Validation Studies}

For validation, we used results from two studies. The first one was an EWAS of CpGs associated with mortality [19]. The study population was made up of the Lothian Birth Cohorts LBC1921 and LBC1936 [20], consisting of individuals born in $1921(N=550)$ and $1936(N=1,091)$, respectively. Blood samples were taken in 1999 and 2004, and vital status obtained in 2013 (454 deaths) and 2011 (186 deaths), respectively. Descriptions of the cohorts and methylation data can be found in [7]. Adjustment for cell type composition was performed using the R-package celltypes 450.

The second study was from the Swedish Adoption/Twin Study of Aging (SATSA) [21] and included methylation and survival data on 385 individuals aged 48-99 at baseline, of which 231 died before follow-up (up to 20 years). The sample included 73 monozygotic and 96 dizygotic twin pairs. The cell type composition was estimated using the Houseman method [22] and correction for batch effects was done using the ComBat function from the R-package sva [23].

\subsection{Statistical Analysis}

All analyses below used methylation $\beta$ values corrected for batch effects and cell type composition, and were standardized to a standard deviation of one. In the analyses of the Danish twins, we adjusted for the three different cohorts.

\subsubsection{Univariate Analysis}

The association between methylation of individual $\mathrm{CpG}$ sites and time from blood sample to death was analyzed in the Danish twin data using a Cox frailty model allowing each twin pair to have a unique gamma-distributed hazard ratio modeling pair-specific weaknesses (e.g., genetic or environmental). The model was adjusted for sex, age, and cohort, and the results were corrected for multiple testing by the Benjamini-Hochberg procedure. The Cox proportional hazards assumption was assessed using martingale and Shoenfeld residuals. For CpG sites identified by this 
procedure, a matched intra-pair comparison of the twins was performed using a stratified Cox model (with pair-specific baseline hazards) in order to control for confounding effects.

\subsubsection{Construction of the Predictor}

To construct a predictor for survival, we applied the least absolute shrinkage and selection operator (LASSO) in a Cox model using the glmnet package in R in order to select a smaller set of CpG sites that predict survival. The LASSO was forced to include sex, age, and cohort in the model, while the twin structure was ignored for this analysis.

Since the LASSO requires complete observations for all sites and each CpG had a small number of missing values ( 0.78 missing on average), we used a single imputation, where each missing value was replaced by its expected value in a linear regression of methylation degree on sex, age, cohort, and three principal components.

To make the LASSO output less sensitive to the specific choice of sample, we applied stability selection [24] modified as suggested in [25]. Thus, we ran 1000 replications of the LASSO on randomly chosen subsets of our sample with half the sample size. In each replication, the smoothness parameter was chosen as the minimizer of the partial likelihood under 10-fold cross-validation plus one half standard error. This was an ad hoc choice made to ensure a reasonably sized predictor. The CpGs selected in more than $80 \%$ of the replications were chosen for the final model.

Fitting a Cox model with sex, age, and the sites chosen by the LASSO, we used the log hazard ratio as a predictor for mortality. The estimated coefficients were used for predictions in the two validation datasets.

\subsubsection{Heritability}

To get an upper bound on the heritability of DNA methylation for the CpG sites found in the analyses above, we computed monozygotic twin correlations of methylation levels after correction for sex, age, and cohort.

\subsubsection{Validation}

The sites discovered in the univariate analysis were validated by fitting a Cox model for each on The Lothian Birth Cohort, LBC, and The Swedish Twin Study of Aging, SATSA, datasets adjusting for sex, age, and twin structure (SATSA). The resulting $p$-values and hazard ratios were compared to those from the Danish dataset. As further validation, we computed a 10-fold cross-validated Harrell's C $[26,27]$ for a Cox model with sex, age, and each CpG site as covariates. This was done both for the Danish, British, and Swedish datasets and compared to a cross-validated Harrell's C for the basic Cox model based on sex and age only. A similar validation was performed for the sites involved in the predictor.

To validate the predictor, an overall Harrell's $C$ for the Cox model with the predictor as the only covariate was computed for all three datasets. Moreover, to investigate whether the effect of the predictor was independent of underlying genetic effects, we conducted an intrapair analysis on the Danish and Swedish twin datasets by fitting a Cox model stratified by the twin pair with the predictor as only covariate. The predictive ability over time of the sites chosen for the predictor was assessed by computing a time-varying area under the ROC curve, AUC, using the timeROC package in R. The AUC was validated by 5-fold cross-validation on the Danish dataset, and rough confidence intervals were constructed by averaging the confidence intervals produced by each fold. Moreover, we computed a time-varying AUC for the predictor on the two validation datasets. Finally, we drew a correlation plot for the methylation levels of the CpG sites chosen for the predictor. 


\subsubsection{Comparison to the Literature}

The sites identified in this study were compared to the CpG sites linked to mortality in previous studies [9-11] and the CpG sites associated with aging in [5,6]. A comparison to the EWAS performed on LBC in [19] can be found in that paper.

\section{Results}

We analyzed survival data for 435 monozygotic twin pairs from three Danish twin studies (LSADT, MADT, and BWD). MADT was the largest study with 482 individuals, while the BWD and LSADT included 150 and 238 individuals, respectively. The participants in the LSADT were generally older (73-90 years) and had longer follow-up times (up to 20 years) compared to those of the MADT and BWD (age 55-79, max 8 years of follow-up); thus the most deaths occurred in the LSADT. In total, 258 participants died before the end of the study. MADT and BWD were homogeneous in gender, while $76 \%$ of the LSADT participants were female. The basic characteristics of the study population are shown in Table 1. DNA methylation levels were measured for all individuals. After pre-processing, methylation data for $441,160 \mathrm{CpG}$ sites was available.

\subsection{Univariate Analyses}

The association between methylation degree at each single CpG site and survival was analyzed by Cox regression adjusting for sex, age, and cohort while taking the twin pairing into account. This resulted in a total of $2806 \mathrm{CpG}$ sites with false discovery rate (FDR) below 0.05. A full list can be found in Supplementary Table S1. For 2074 of the 2806 top sites (73.9\%), hypomethylation was associated with increased mortality corresponding to a hazard ratio (HR) below 1, while hypermethylation was associated with mortality for the remaining 732 sites (HR $>1$ ). Twenty-four CpG sites had an unadjusted $p$-value below $10^{-7}$, corresponding to a Bonferroni corrected $p$-value below 0.044 . These are listed in Table 2. In a matched intra-pair comparison of the twin and co-twin the suggested direction of association was confirmed for all 24 sites and in 16 of the sites the association was significant at a 0.05 level, as can be seen from Table 2 . 
Table 2. The $24 \mathrm{CpG}$ sites with $p<10^{-7}$ in the univariate analysis.

\begin{tabular}{|c|c|c|c|c|c|c|}
\hline CpG Site & HR & $p$ & FDR & Chromosome & Gene Name & MZ Correlation \\
\hline $\operatorname{cg} 07626482^{+}$ & 0.64 & $3.211 \times 10^{-12}$ & $1.42 \times 10^{-6}$ & 19 & SLC1A5 & $0.43^{* * *}$ \\
\hline cg12627844 & 0.69 & $5.20 \times 10^{-9}$ & $1.15 \times 10^{-3}$ & 2 & VPS54 & $0.44^{* * *}$ \\
\hline $\operatorname{cg} 05339037^{\dagger}$ & 0.77 & $1.18 \times 10^{-8}$ & $1.31 \times 10^{-3}$ & 19 & & $0.44^{* * *}$ \\
\hline cg03487027 & 0.73 & $1.40 \times 10^{-8}$ & $1.31 \times 10^{-3}$ & 10 & ZNF503 & $0.51^{* * *}$ \\
\hline cg20162822 & 0.78 & $1.81 \times 10^{-8}$ & $1.31 \times 10^{-3}$ & 17 & SERPINF2 & -0.05 \\
\hline cg25121437 & 0.73 & $2.04 \times 10^{-8}$ & $1.31 \times 10^{-3}$ & 11 & FEZ1 & $0.29 * * *$ \\
\hline $\operatorname{cg} 10589813^{+}$ & 0.71 & $2.76 \times 10^{-8}$ & $1.31 \times 10^{-3}$ & 20 & & $0.41^{* * *}$ \\
\hline $\operatorname{cg} 08627624^{\dagger}$ & 0.69 & $2.85 \times 10^{-8}$ & $1.31 \times 10^{-3}$ & 10 & & -0.04 \\
\hline cg06598544 & 0.78 & $3.13 \times 10^{-8}$ & $1.31 \times 10^{-3}$ & 20 & COL9A3 & $0.31^{* * *}$ \\
\hline $\operatorname{cg} 23591302 * *$ & 0.74 & $3.14 \times 10^{-8}$ & $1.31 \times 10^{-3}$ & 12 & PRICKLE1 & $0.15^{* * *}$ \\
\hline $\operatorname{cg} 13580749^{\dagger}$ & 0.72 & $3.25 \times 10^{-8}$ & $1.31 \times 10^{-3}$ & 9 & & $0.18^{* * *}$ \\
\hline $\operatorname{cg} 15013177^{*, * *,+}$ & 0.77 & $3.98 \times 10^{-8}$ & $1.31 \times 10^{-3}$ & 3 & CNTN6 & $0.12 * * *$ \\
\hline $\operatorname{cg} 17087741^{* *,+}$ & 0.71 & $4.16 \times 10^{-8}$ & $1.31 \times 10^{-3}$ & 2 & & $0.45^{* * *}$ \\
\hline $\operatorname{cg} 04716447^{* *+\dagger}$ & 1.42 & $4.16 \times 10^{-8}$ & $1.31 \times 10^{-3}$ & 12 & & $0.18^{* * *}$ \\
\hline $\operatorname{cg} 05232694^{+}$ & 0.72 & $4.64 \times 10^{-8}$ & $1.33 \times 10^{-3}$ & 20 & & $0.31^{* * *}$ \\
\hline $\operatorname{cg} 06172950^{\dagger}$ & 0.74 & $4.81 \times 10^{-8}$ & $1.33 \times 10^{-3}$ & 13 & COG3 & $0.27^{* * *}$ \\
\hline $\operatorname{cg} 26186132^{\dagger}$ & 0.71 & $5.66 \times 10^{-8}$ & $1.41 \times 10^{-3}$ & 6 & C6orf147 & -0.07 \\
\hline cg11339912 & 0.72 & $5.74 \times 10^{-8}$ & $1.41 \times 10^{-3}$ & 5 & SH3RF2 & $0.39^{* * *}$ \\
\hline $\operatorname{cg} 24750887^{\dagger}$ & 1.42 & $6.22 \times 10^{-8}$ & $1.44 \times 10^{-3}$ & 4 & HERC3 & 0.05 \\
\hline $\operatorname{cg} 15763258^{\dagger}$ & 0.73 & $6.52 \times 10^{-8}$ & $1.44 \times 10^{-3}$ & 11 & FLI1 & $0.36^{* * *}$ \\
\hline $\operatorname{cg} 15871086^{+}$ & 0.74 & $7.65 \times 10^{-8}$ & $1.61 \times 10^{-3}$ & 18 & & $0.36^{* * *}$ \\
\hline $\operatorname{cg} 02657160^{\dagger}$ & 0.70 & $8.81 \times 10^{-8}$ & $1.72 \times 10^{-3}$ & 3 & CPOX & $0.18^{* * *}$ \\
\hline $\operatorname{cg} 02711608^{* *, \dagger}$ & 0.69 & $8.99 \times 10^{-8}$ & $1.72 \times 10^{-3}$ & 19 & SLC1A5 & $0.48^{* * *}$ \\
\hline $\operatorname{cg} 17459949^{\dagger}$ & 0.73 & $9.79 \times 10^{-8}$ & $1.80 \times 10^{-3}$ & 10 & & -0.06 \\
\hline
\end{tabular}

FDR: false discovery rate. ${ }^{*}$ Polymorphic site according to [16]. ${ }^{* *}$ Single nucleotide polymorphism (SNP) within-probe binding region (according to annotation file). ${ }^{* * *}$ Significant at the 0.05 level. + A significant hazard ratio (HR) at 0.05 level in intra-pair comparison.

For each of the 24 top CpG sites, Table 3 shows the results of a similar Cox analysis on the validation datasets. Comparing the $p$-values and direction of the hazard ratios, seven CpGs could be validated in the Lothian Birth Cohort, namely cg07626482, cg05339037, cg10589813, cg17087741, cg11339912, cg15871086, and cg02711608. For all seven sites, hypomethylation was associated with mortality. In the Swedish dataset, 5 of the 24 CpGs were missing. The remaining 19 sites did not show any convincing effect when multiple testing was taken into account. However, the three lowest $p$-values belong to three of the seven sites validated in the LBC (cg07626482, cg05339037, and cg10589813). 
Table 3. Hazard ratio and association $p$-value for the top 24 sites in a Cox frailty model adjusted for sex, age, and twin structure in the discovery and validation datasets. Five CpGs were not available in the Swedish data.

\begin{tabular}{|c|c|c|c|c|c|c|}
\hline \multirow{2}{*}{ CpG Site } & \multicolumn{2}{|c|}{ Danish Twins } & \multicolumn{2}{|c|}{ Lothian Birth Cohort } & \multicolumn{2}{|c|}{ Swedish Twins } \\
\hline & HR & $p$ & HR & $p$ & HR & $p$ \\
\hline $\operatorname{cg} 07626482$ & 0.64 & $3.21 \times 10^{-12}$ & 0.55 & $3.18 \times 10^{-3}$ & 0.88 & 0.09 \\
\hline $\operatorname{cg} 12627844$ & 0.69 & $5.20 \times 10^{-9}$ & 0.94 & 0.53 & - & - \\
\hline cg05339037 & 0.77 & $1.18 \times 10^{-8}$ & 0.42 & $1.70 \times 10^{-4}$ & 0.85 & 0.02 \\
\hline cg03487027 & 0.73 & $1.40 \times 10^{-8}$ & 1.09 & $4.81 \times 10^{-3}$ & 0.99 & 0.87 \\
\hline cg20162822 & 0.78 & $1.81 \times 10^{-8}$ & 0.96 & 0.64 & 1.08 & 0.31 \\
\hline cg25121437 & 0.73 & $2.04 \times 10^{-8}$ & 0.91 & 0.48 & 1.07 & 0.30 \\
\hline $\operatorname{cg} 10589813$ & 0.71 & $2.76 \times 10^{-8}$ & 0.56 & $2.71 \times 10^{-3}$ & 0.85 & 0.03 \\
\hline cg08627624 & 0.69 & $2.85 \times 10^{-8}$ & 1.08 & 0.48 & - & - \\
\hline $\operatorname{cg} 06598544$ & 0.78 & $3.13 \times 10^{-8}$ & 1.06 & 0.13 & 1.04 & 0.57 \\
\hline cg23591302 & 0.74 & $3.14 \times 10^{-8}$ & 1.05 & 0.11 & - & - \\
\hline $\operatorname{cg} 13580749$ & 0.72 & $3.25 \times 10^{-8}$ & 1.00 & 1.00 & - & - \\
\hline cg15013177 & 0.77 & $3.98 \times 10^{-8}$ & 1.09 & 0.42 & 0.97 & 0.66 \\
\hline $\operatorname{cg} 17087741$ & 0.71 & $4.16 \times 10^{-8}$ & 0.57 & $3.22 \times 10^{-7}$ & - & - \\
\hline cg04716447 & 1.42 & $4.16 \times 10^{-8}$ & 1.37 & 0.15 & 1.06 & 0.47 \\
\hline cg05232694 & 0.72 & $4.64 \times 10^{-8}$ & 0.86 & 0.07 & 0.90 & 0.17 \\
\hline cg06172950 & 0.74 & $4.81 \times 10^{-8}$ & 0.77 & 0.034 & 0.98 & 0.75 \\
\hline cg26186132 & 0.71 & $5.66 \times 10^{-8}$ & 1.18 & 0.33 & 1.10 & 0.17 \\
\hline $\operatorname{cg} 11339912$ & 0.72 & $5.74 \times 10^{-8}$ & 0.65 & $2.27 \times 10^{-3}$ & 0.91 & 0.18 \\
\hline cg24750887 & 1.42 & $6.22 \times 10^{-8}$ & 1.05 & 0.52 & 1.02 & 0.82 \\
\hline $\operatorname{cg} 15763258$ & 0.73 & $6.52 \times 10^{-8}$ & 0.70 & 0.03 & 0.97 & 0.69 \\
\hline cg15871086 & 0.74 & $7.65 \times 10^{-8}$ & 0.66 & $6.82 \times 10^{-3}$ & 0.97 & 0.71 \\
\hline cg02657160 & 0.70 & $8.81 \times 10^{-8}$ & 1.01 & 0.93 & 0.90 & 0.13 \\
\hline cg02711608 & 0.69 & $8.99 \times 10^{-8}$ & 0.53 & $5.30 \times 10^{-5}$ & 0.99 & 0.94 \\
\hline $\operatorname{cg} 17459949$ & 0.73 & $9.79 \times 10^{-8}$ & 1.07 & 0.11 & 0.97 & 0.68 \\
\hline
\end{tabular}

As a second validation, we computed a cross-validated Harrell's $C$ for the Cox model including sex, age, and each of the 24 top sites. Harrell's C measures the concordance between survival time and the hazard ratio computed from sex, age, and methylation degree. The result is shown in Figure 1 and compared to the model with sex and age only. For the Danish twin data, each CpG site improves the basic model. Most sites also improve concordance in the Lothian Birth Cohort although the magnitude of improvement is smaller. The best validated sites are the seven sites also validated by $p$-values together with cg06172950 and cg15763258 (excluding sites with inconsistent hazard ratio). The 19 sites available in SATSA generally show a poor performance. The only three sites showing moderately good performance are the same as the three best ones based on $p$-values. 

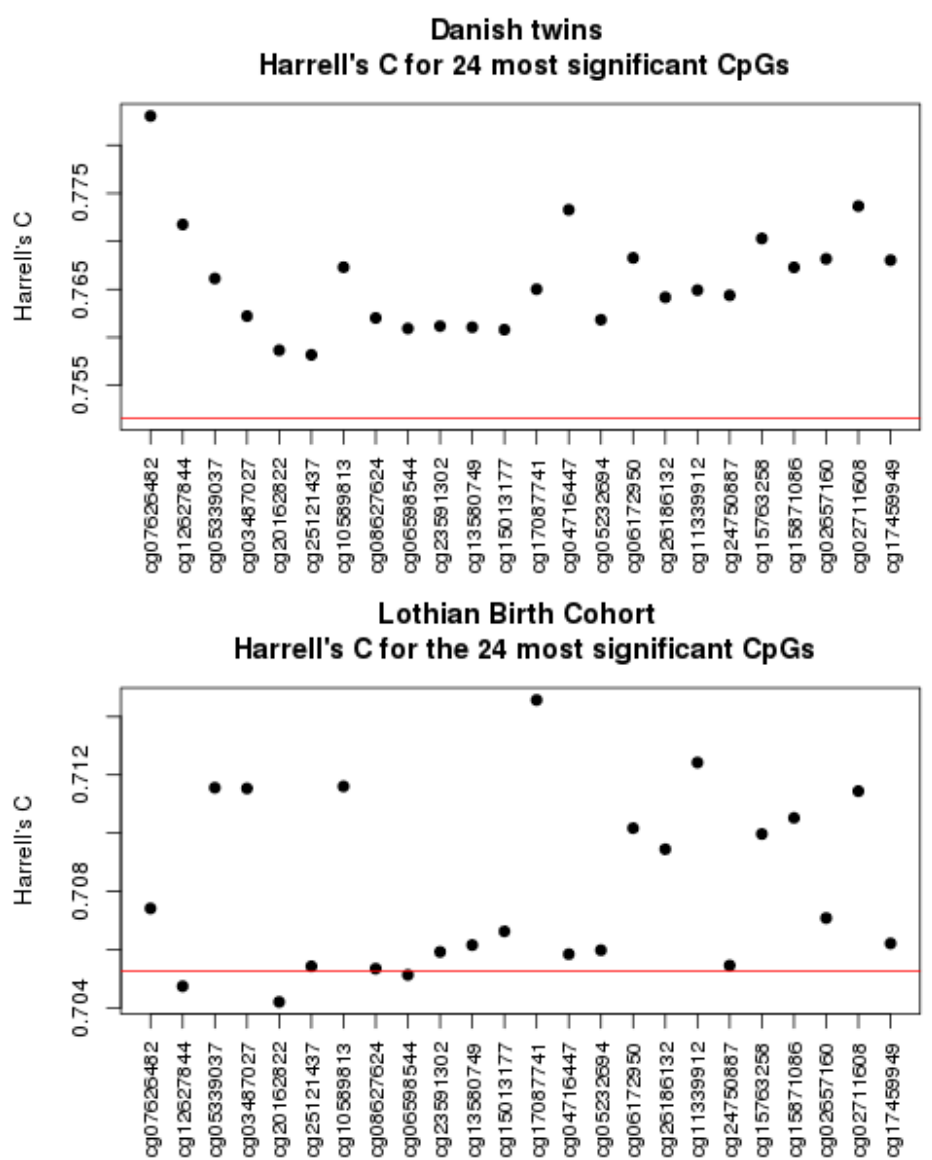

Swedish twins

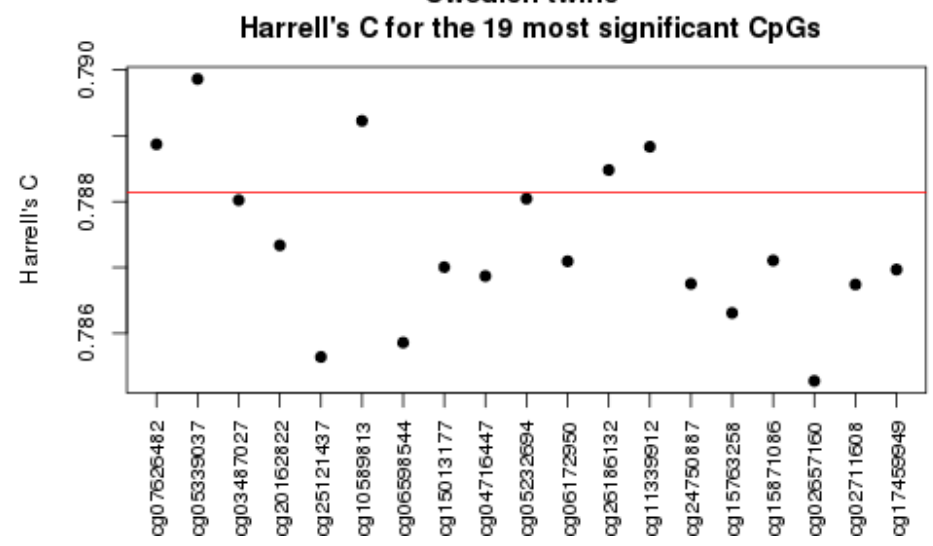

Figure 1. The 10-fold cross-validated Harrell's C for the 24 most significant sites in the univariate analysis. Sex, age, and cohort are included in the model. The red line corresponds to the model based on sex, age, and cohort only.

There were no obvious violations of the proportional hazards assumption, but some outliers in the methylation data may have influenced the results. An intrapair analysis was not feasible due to sparse information from discordant pairs.

\subsection{Mortality Predictor}

We used LASSO regression to choose 14 CpG sites for a mortality predictor. These sites are listed in Table 4. The predictor is defined as a linear combination of sex, age, and methylation values at the 14 sites with the coefficients given in Table 4. 
Table 4. The variables chosen by the least absolute shrinkage and selection operator (LASSO) for the mortality predictor. The second column gives the coefficients for the linear predictor. All methylation values are normalized to have a standard deviation of 1 .

\begin{tabular}{|c|c|c|c|c|c|c|}
\hline Covariate & Coefficient $* *$ & $\mathrm{SE}^{* *}$ & $p^{* *}$ & Chromosome & Gene Name & MZ Correlation \\
\hline $\operatorname{cg} 02537149$ & 0.158 & 0.0664 & 0.0173 & 1 & LRRC41, UQCRH & 0.00 \\
\hline $\operatorname{cg} 02691019$ & 0.0906 & 0.0564 & 0.108 & 16 & & -0.07 \\
\hline $\operatorname{cg} 04716447 *$ & 0.308 & 0.0623 & $7.36 \times 10^{-7}$ & 12 & & $0.18^{* * *}$ \\
\hline cg05232694 & -0.155 & 0.0686 & 0.0234 & 20 & & $0.31^{* * *}$ \\
\hline cg07626482 & -0.160 & 0.0820 & 0.0508 & 19 & SLC1A5 & $0.43^{* * *}$ \\
\hline cg12880095 & 0.115 & 0.0621 & 0.0650 & 17 & & $0.53^{* * *}$ \\
\hline $\operatorname{cg} 14304264$ & 0.140 & 0.0705 & 0.0467 & 15 & МСТP2 & 0.00 \\
\hline cg17459949 & -0.176 & 0.0640 & $5.95 \times 10^{-3}$ & 10 & & -0.06 \\
\hline cg20164226* & 0.0579 & 0.0579 & 0.317 & 7 & & $0.27^{* * *}$ \\
\hline cg21381949 & -0.112 & 0.0592 & 0.0585 & 3 & LEPREL1 & $0.12^{* * *}$ \\
\hline cg22304262 & -0.273 & 0.0822 & $8.97 \times 10^{-4}$ & 19 & SLC1A5 & $0.38^{* * *}$ \\
\hline cg24750887 & 0.205 & 0.0663 & $1.96 \times 10^{-3}$ & 4 & HERC3 & 0.05 \\
\hline cg24967142 & 0.219 & 0.0669 & $1.04 \times 10^{-3}$ & 12 & C12orf47, MAPКАРК5 & -0.08 \\
\hline $\operatorname{cg} 26186132$ & -0.228 & 0.0688 & $9.11 \times 10^{-4}$ & 6 & C6orf147 & -0.07 \\
\hline Sex (female) & -0.702 & 0.140 & $5.71 \times 10^{-7}$ & & & \\
\hline Age (in years) & 0.139 & 0.0165 & $<2 \times 10^{-16}$ & & & \\
\hline
\end{tabular}

${ }^{*}$ SNP within the probe-binding region. ${ }^{* *}$ Coefficients, standard error, and $p$-value in a Cox model fitted with all 14 sites, sex, and, age. ${ }^{* * *}$ Significant at the 0.05 level.

The individual associations of the 14 sites with mortality were validated using Harrell's C exactly as in the univariate analysis (see Supplementary Figure S1). They again performed well on the Danish twin data. However, we remark that stability selection serves as a sort of cross-validation in the selection process, and hence the resulting sites can be expected to perform well under cross-validation. Most of the 14 sites could be validated on the LBC data, while the SATSA data again showed poor concordance with survival for the 12 sites available in the dataset. The LASSO tended to choose sites that are uncorrelated. This was confirmed by Supplementary Figure S2, showing negligible correlations between most of the 14 sites. The only exceptions are cg07626482 and $\operatorname{cg} 22304262$, which are both located at the gene SLC1A5.

As an overall assessment of the predictive ability of the mortality predictor, we computed a Harrell's $C$ on the Danish twin data. This yielded $C=0.85$ both with and without 10-fold cross-validation, which was an improvement compared to the model based on sex and age only $(C=0.75)$. However, for the LBC and SATSA datasets, we obtained $C=0.69$ and $C=0.75$, respectively, which was in fact worse than the model with sex and age only $(C=0.70$ and $C=0.78$, respectively).

Figure 2 shows a time-varying AUC measuring the ability of the predictor to predict survival a given number of years after the initial blood sample. It shows a good and time-stable performance in the Danish dataset and is clearly better than the predictor based on sex and age only at all time points. However, the validations on LBC and SATSA do not show any improvement of the standard predictor based on sex and age.

To confirm that the observed effect of the predictor on the Danish data was independent of underlying genetic variation, we conducted an intrapair analysis measuring the predictive ability within twin pairs. We found a highly significant effect of thelpredictor $\left(p=2.91 \cdot 10^{-8}\right)$. Since monozygotic twins are matched on the underlying genes, this shows that the effect of the predictor cannot be due to underlying genetic effects alone. A similar analysis on the SATSA data only obtained $p=0.097$. 


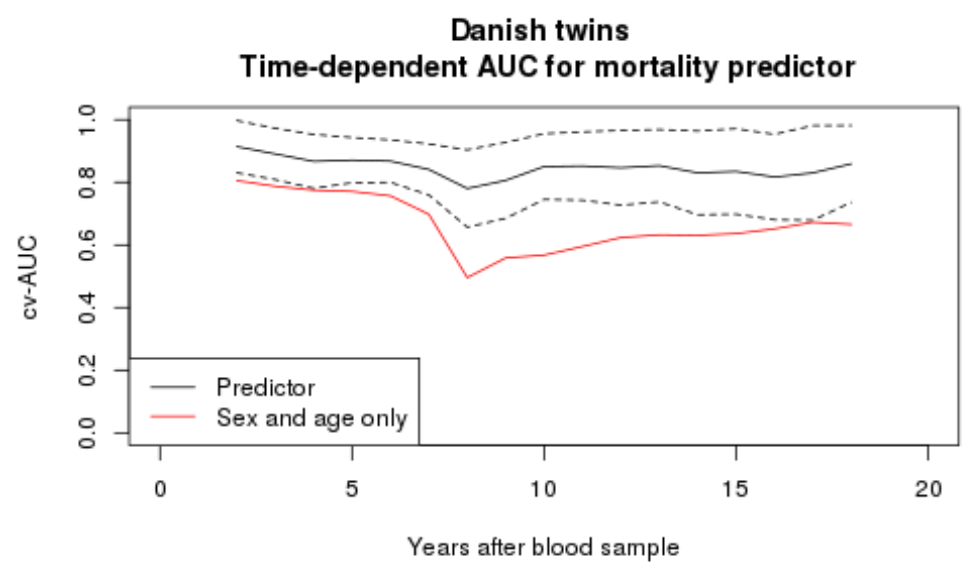

Lothian Birth Cohort

Time-dependent AUC for mortality predictor

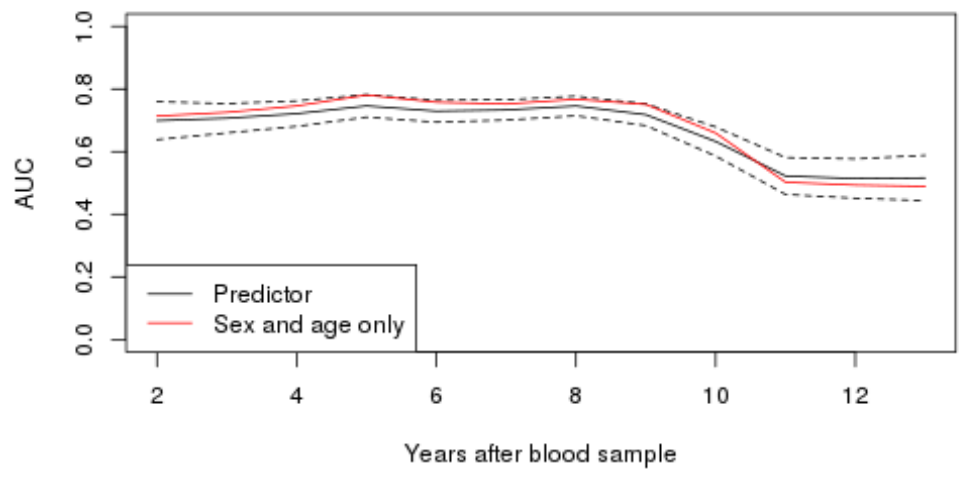

Swedish twins

Time-dependent AUC for mortality predictor

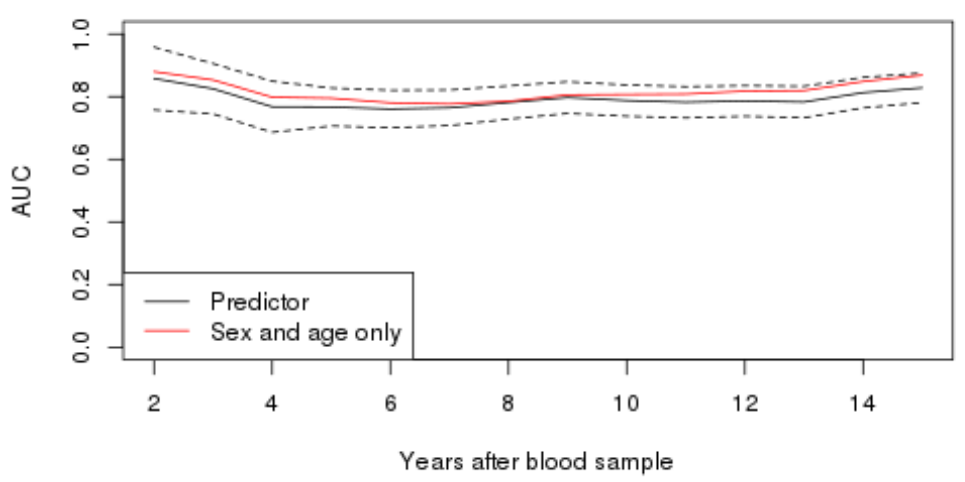

Figure 2. The 10-fold cross-validated time-varying AUC for the LASSO predictor based on all 14 CpG sites, sex, and age. This is compared to the model based on sex and age only.

\subsection{Heritability}

The last columns of Tables 2 and 4 show the correlation between methylation measurements within monozygotic twin pairs for the top 24 sites and the 14 sites in the predictor, respectively, providing an upper bound on the heritability. The correlation highly varies between different CpGs. For some sites, the correlation is essentially 0, while for others, the correlation is as high as 0.53 , indicating some heritability or shared environmental effects. It is worth noting that the seven best validated sites in top 24 all have rather high correlations (0.36-0.45) allowing for a potentially high heritability of the mortality-related methylation pattern. 


\subsection{Comparison to Other Studies}

In [9], a Cox analysis of data from the Germanl ESTHER study was used to identify 11,063 mortality-related CpG sites (FDR $\leq 0.05)$. These were boiled down to 58 using LASSO regression in a validation dataset. Two of the top 24 sites from our univariate analysis overlapped with these 58, namely cg07626482 and cg02657160, having the 1st and 22nd lowest $p$-values in our study, respectively. Out of the 58 sites, 19 (33\%) had FDR $\leq 0.05$ in our study, and 35 (60\%) had an unadjusted $p$-value below 0.05 in our study. Ten sites were selected for a final mortality predicting model in [9], of which seven $(70 \%)$ had $p \leq 0.05$. A list of the 35 sites with $p \leq 0.05$ in our study can be found in Supplementary Table S2. For all 35 sites, the hazard ratios were consistent in the two studies, and in 31 cases $(89 \%)$ hypomethylation was associated with mortality.

Data from the Finnish Vitality 90+ study was analyzed in [10]. With 2.55 years of follow-up, 19 mortality-related sites were found (FDR $\leq 0.5$ ). None of these overlapped with our 2806 sites and only one $(5.2 \%)$ had $p \leq 0.05$ (cg08596308, $p=0.021)$. The average $p$-value for the 19 sites was 0.55 . Thus, the $p$-values are not lower than what would be expected by chance. With 4-year follow-up data, seven sites with FDR $\leq 0.5$ were found in [10]. Again, only cg08596308 reached significance in our study.

In [11], data from the Italian InCHIANTI study was analyzed. Methylation at 88 sites associated with mortality $(p \leq 0.001)$ was listed in the paper. One of these had FDR $\leq 0.05$ (cg00522231, $\left.\mathrm{HR}=1.34, p=2.5 \cdot 10^{-4}\right)$ in our study, while $21(24 \%)$ had $p \leq 0.05$.

Horvath's methylation age [5] was based on methylation of $353 \mathrm{CpG}$ sites. One of these had FDR $\leq 0.05$ in our study $\left(\operatorname{cg} 01485645, p=1.4 \cdot 10^{-5}\right)$. In total, 35 of the sites $(10 \%)$ showed significance. The methylation age defined by Hannum [6] is based on $71 \mathrm{CpG}$ sites. None of these are among our top 2806 sites, while $12(17 \%)$ have $p \leq 0.05$.

\section{Discussion}

We analyzed DNA methylation data on 870 monozygotic twins to find CpG sites linked to mortality. We found $2806 \mathrm{CpG}$ sites associated with mortality (FDR $\leq 0.05$ ) indicating an abundance of mortality-related CpGs that is supported by [9,19]. We identified 24 sites with a $p$-value less than $10^{-7}$, and out of these, cg07626482, cg05339037, cg10589813, cg17087741, cg11339912, cg15871086, and cg02711608 were validated in an independent dataset, while cg02657160 had been reported in an earlier mortality study [9]. For all eight sites, hypomethylation was associated with increased mortality, and for the first seven sites, MZ correlation ranged from 0.36 to 0.45 , indicating an inherited or shared environmental contribution to the methylation of mortality-related sites. The seven best validated sites in the top 24 all had rather high correlations, allowing for a potentially high heritability of mortality-related methylation patterns that is novel and should be pursued in further studies.

In order to reduce the influence of confounders, we compared MZ twins to their co-twin. This basically enables us to study to which degree the twin with the highest methylation value is also the twin in the pair with longest survival. This is a very strong test for association. For all 24 identified, the suggested direction of the CpG survival association was confirmed, and for 16 sites, the $p$-value was below 0.05 .

The most significant CpG site in the univariate analysis was cg07626482, which also had the most extreme hazard ratio among the top 2806 sites and the best cross-validated Harrell's C among the top 24 sites. Moreover, it was chosen by the LASSO in all 1000 replications of the stability selection. This was confirmed in the Lothian Birth Cohort and supported by the Swedish twin data. Interestingly, it was also among the 58 sites reported in [9]. This CpG site is located at the shore of a CpG Island in the 5 ' region of the SLC1A5 gene. The surrounding region contains an enrichment of histone marks and transcription factor binding sites, which may suggest a regulatory role for this region. The SLC1A5 gene encodes a sodium-dependent neutral amino acid transporter [28]. High expression of SLC1A5 has been associated with poor prognosis for various types of cancer, e.g., in [29-31]. Another CpG site among our top 24 sites was located at this gene, namely cg02711608, which was also validated in the LBC. Hypomethylation of this specific site has been associated with type 2 diabetes [32]. In total, three 
of the 26 most significant sites in our study, two of the sites in the LASSO predictor, and three of the 58 CPG sites in [9] were located at SLC1A5, strongly indicating that hypomethylation of this region is associated with increased mortality.

The second most significant site, $\operatorname{cg} 12627844$, is located at the shore of a CpG Island in the $5^{\prime}$ region of the VPS54 gene. Again, the presence of nearby histone mark enrichment, areas of DNA hypersensitivity, and transcription factor binding sites suggests that this is a regulatory region. This was not validated in the Lothian Birth Cohort and the information was not available in the Swedish dataset.

The third site in Table 2, cg05339037, was validated in the LBC and supported by SATSA. It is located to a shelf in the vicinity of a probable non-coding RNA gene of the miR-3074 gene family. The region is characterized by a high level of enrichment of the H3K27Ac histone mark, as well as DNase hypersensitivity, and numerous possible transcription factor recognition sites. This all points to the presence of a promoter.

In addition, the seventh site in Table 2, cg10589813, replicates in LBC and is supported by SATSA. It is located in the south shore of a CpG island, which lies near the CEBPB gene, encoding an important transcription factor involved in the regulation of the expression of genes involved in immune and inflammatory responses [33-35].

The 18th site, cg11339912, was also confirmed by the LBC. It is located at an intron of the SH3RF2 gene. The gene is highly expressed in cancer tissue [36]. We did not encounter any relevant information on cg17087741, cg15871086, or cg02657160.

We also constructed a predictor for mortality. It showed good performance on the Danish dataset, and also under cross-validation and when correcting for at least genetic effects by MZ intra-pair comparison. It did not seem to lose predictive power during the follow-up period. However, it was not possible to validate it in any of the two independent datasets. This may be because the predictor is sensitive to latent cohort effects (see below) and the various steps taken in the data preprocessing phase. If a predictor is going to be used for predictions across samples in the future, it should come with a manual for data preprocessing and batch effect removal. Alternatively, one might take an approach that is more robust to noise, as in [9]. Including more sites in the predictor, as is done for the age predictors of $[5,6]$, might also make it more robust against noise within a single site and account for more possible causes of death.

While our results were confirmed by the Lothian Birth Cohort to the degree that one might expect, we had problems validating the predictor in the Swedish twin data. This is surprising, as the two studies are very similar, both using samples of Scandinavian twins of comparable size, age span, and follow-up time. Again, this points to the problem of comparing methylation data across different studies, e.g., different preprocessing strategies (see also discussion below on the phenotype in question).

We were able to confirm many of the findings from [9]. This study benefits from a large sample size of twin pairs and an independent dataset used for validation. Moreover, a long list of confounders is controlled for in the validation phase. On the other hand, adjusting for confounders risks overlooking CpGs that e.g., mediate the effect of lifestyle factors or cause preexisting diseases. We did not control for such confounders in the present study. However, the finding that the identified sites to a large degree persist in the intra-pair comparison test, and are vulnerable to only non-shared non-genetic confounders, is highly indicative of an association. The intra-pair comparison is not feasible for the all the EWAS but adds validity to the candidate findings.

The sites found in $[10,11]$ were not as convincingly validated in our dataset. In both cases, this may be due to low power in those studies (36 and 79 deaths, respectively) or differences in the study population. The individuals in [10] were all nonagenarians (aged 90+), and mechanisms causing deaths in very old people might be different from those present in our sample population. Moreover, both studies have much shorter follow-up times. It is possible that sites predicting mortality within the near future are different from those predicting long-term survival. 
Finally, in contrast to EWAS on age-dependent epigenetic changes that reported high replication rates across samples [37], EWAS on mortality deal with an outcome variable (i.e., death) that is subject to more uncertainty than an individual age. Low overlap across studies can be expected due to power limitations across studies. A systematic meta-analysis or a combined analysis would be warranted to justify findings across studies.

Although deviations between chronological and epigenetic age have been observed to predict all-cause mortality [7,8], the CpG sites defining the methylation ages by Horvath and Hannum were generally not strongly associated with mortality in this study. Similar observations were made in previous studies of methylation and mortality (see the discussion sections in [9-11]). A possible explanation may be that sites are chosen for epigenetic clocks because of rather stable age-related changes in methylation independent of diseases, life-style factors, and individual aging patterns. The present contribution may be seen as an initial step among many in explaining systematic patterns of genetic and epigenetic causes that exist [38] for the phenotype of age at death, which is subject to considerable uncertainty.

Supplementary Materials: The following are available online at www.mdpi.com/2073-4425/9/2/78/s1, Figure S1: Cross-validtaed Harrell's C for the $14 \mathrm{CpG}$ sites used for the mortality predictor in the discovery and validation datasets, Figure S2: Correlations between the $14 \mathrm{CpG}$ sites entering the mortality predictor, Table S1: A list of the all 2806 CpG sites with FDR $<0.05$ in the present study including information from annotation file, Table S2: List of all 35 CpG sites dicovered in [9] and showing significance in the present study.

Acknowledgments: The Danish Aging Research Center is supported by a grant from the VELUX Foundation. The study was supported by the European Union's Seventh Framework Programme (FP7/2007-2011) under grant agreement no. 259679, the National Program for Research Infrastructure 2007 from the Danish Agency for Science, Technology and Innovation and the US National Institutes of Health (P01 AG08761), and the Danish Council for Independent Research (DFF-6110-00016). The authors would also like to thank Birgit Debrabant for the cell-type estimates in the Danish cohort. This validation using the LBC study was supported by Velux Foundation research grant \# 000121540. Phenotype collection in the Lothian Birth Cohort 1921 was supported by the UK's Biotechnology and Biological Sciences Research Council (BBSRC), the Royal Society, and the Chief Scientist Office of the Scottish Government. Phenotype collection in the Lothian Birth Cohort 1936 was supported by Age UK (The Disconnected Mind project). Methylation typing was supported by Centre for Cognitive Ageing and Cognitive Epidemiology (Pilot Fund award), Age UK, the Wellcome Trust Institutional Strategic Support Fund, the University of Edinburgh, and the University of Queensland. REM and IJD are members of the University of Edinburgh Centre for Cognitive Ageing and Cognitive Epidemiology (CCACE), which is supported by funding from the BBSRC, the Medical Research Council (MRC), and the University of Edinburgh as part of the cross-council Lifelong Health and Wellbeing initiative (MR/K026992/1). The SATSA study was supported by NIH grants R01 AG04563, AG10175, and AG028555, the MacArthur Foundation Research Network on Successful Aging, the Swedish Council for Working Life and Social Research (FAS/FORTE) (97:0147:1B, 2009-0795, 2013-2292), the Swedish Research Council (825-2007-7460, 825-2009-6141, 521-2013-8689, 2015-03255), the Karolinska Institutet Delfinansiering (KID) grant for doctoral students (Y.W.), the KI Foundation, the Strategic Research Program in Epidemiology at Karolinska Institutet, and by Erik Rönnbergs donation for scientific studies in aging and age-related diseases.

Author Contributions: J.H. and L.C. initiated and planned the study; L.C. and K.C. provided data for the main analysis; N.L.H., S.H., R.E.M., I.J.D. provided the validation data; M.S. prepared data for analysis, A.M.S. and J.H. conducted the main data analysis, J.L., Q.T., Y.W., and S.H. analyzed the validation data, A.M.S., J.H., and L.C. wrote the paper.

Conflicts of Interest: The authors declare no conflict of interest.

\section{References}

1. Volkmar, M.; Dedeurwaerder, S.; Cunha, D.A.; Ndlovu, M.N.; Defrance, M.; Deplus, R.; Calonne, E.; Volkmar, U.; Igoillo-Esteve, M.; Naamane, N.; et al. DNA methylation profiling identifies epigenetic dysregulation in pancreatic islets from type 2 diabetic patients. EMBO J. 2012, 31, 1405-1426.

2. Dawson, M.A.; Kouzarides, T. Cancer epigenetics: From mechanism to therapy. Cell 2012, 150, 12-27.

3. Webster, A.L.; Yan, M.S.; Marsden, P.A. Epigenetics and cardiovascular disease. Can. J. Cardiol. 2013, 29, $46-57$.

4. Kramer, J.M. Epigenetic regulation of memory: implications in human cognitive disorders. Biomol. Concepts 2012, 4, 1-12.

5. Horvath, S. DNA methylation age of human tissues and cell types. Genome Biol. 2013, 14, 3156. 
6. Hannum, G.; Guinney, J.; Zhao, L.; Zhang, L.; Hughes, G.; Sadda, S.; Klotzle, B.; Bibikova, M.; Fan, J.B.; Gao, Y.; et al. Genome-wide methylation profiles reveal quantitative views of human aging rates. Mol. Cell 2013, 49, 359-367.

7. Marioni, R.E.; Shah, S.; McRae, A.F.; Chen, B.H.; Colicino, E.; Harris, S.E.; Gibson, J.; Henders, A.K.; Redmond, P.; Cox, S.R.; et al. DNA methylation age of blood predicts all-cause mortality in later life. Genome Biol. 2015, 16, doi:10.1186/s13059-015-0584-6.

8. Christiansen, L.; Lenart, A.; Tan, Q.; Vaupel, J.W.; Aviv, A.; McGue, M.; Christensen, K. DNA methylation age is associated with mortality in a longitudinal Danish twin study. Aging Cell 2016, 15, 149-154.

9. Zhang, Y.; Wilson, R.; Heiss, J.; Breitling, L.P.; Saum, K.-U.; Schöttker, B.; Holleczek, B.; Waldenberger, M.; Peters, A.; Brenner, H. DNA methylation signatures in peripheral blood strongly predict all-cause mortality. Nat. Commun. 2017, 8, 14617.

10. Jylhävä, J.; Kananen, L.; Raitanen, J.; Marttila, S.; Nevalainen, T.; Hervonen, A.; Jylhä, M.; Hurme, M. Methylomic predictors demonstrate the role of NF- $\kappa \mathrm{B}$ in old-age mortality and are unrelated to the aging-associated epigenetic drift. Oncotarget 2016, 7, 19228-19241.

11. Moore, A.Z.; Hernandez, D.G.; Tanaka, T.; Pilling, L.C.; Nalls, M.A.; Bandinelli, S.; Singleton, A.B.; Ferrucci, L. Change in Epigenome-Wide DNA Methylation Over 9 Years and Subsequent Mortality: Results From the InCHIANTI Study. J. Gerontol. A Biol. Sci. Med. Sci. 2016, 71, 1029-1035.

12. Frost, M.; Petersen, I.; Brixen, K.; Beck-Nielsen, H.; Holst, J.J.; Christiansen, L.; Højlund, K.; Christensen, K. Adult glucose metabolism in extremely birthweight-discordant monozygotic twins. Diabetologia 2012, 55, 3204-3212.

13. Christensen, K.; Holm, N.V.; Mcgue, M.; Corder, L.; Vaupel, J.W. A Danish Population-Based Twin Study on General Health in the Elderly. J. Aging Health 1999, 11, 49-64.

14. Gaist, D.; Bathum, L.; Skytthe, A.; Jensen, T.K.; McGue, M.; Vaupel, J.W.; Christensen, K. Strength and anthropometric measures in identical and fraternal twins: no evidence of masculinization of females with male co-twins. Epidemiology 2000, 11, 340-343.

15. Tobi, E.W.; Slieker, R.C.; Stein, A.D.; Suchiman, H.E.D.; Slagboom, P.E.; van Zwet, E.W.; Heijmans, B.T.; Lumey, L.H. Early gestation as the critical time-window for changes in the prenatal environment to affect the adult human blood methylome. Int. J. Epidemiol. 2015, 44, 1211-1223.

16. Chen, Y.-A.; Lemire, M.; Choufani, S.; Butcher, D.T.; Grafodatskaya, D.; Zanke, B.W.; Gallinger, S.; Hudson, T.J.; Weksberg, R. Discovery of cross-reactive probes and polymorphic CpGs in the Illumina Infinium HumanMethylation450 microarray. Epigenetics 2013, 8, 203-209.

17. Fortin, J.-P.; Labbe, A.; Lemire, M.; Zanke, B.W.; Hudson, T.J.; Fertig, E.J.; Greenwood, C.M.T.; Hansen, K.D. Functional normalization of $450 \mathrm{k}$ methylation array data improves replication in large cancer studies. Genome Biol. 2014, 15, 503.

18. Debrabant, B.; Sørensen, M.; Christiansen, L.; Tan, Q.; McGue, M.; Christensen, K.; Hjelmborg, J. DNA methylation age and perceived age in elderly Danish twins. Mech. Ageing Dev. 2017, doi:10.1016/j.mad.2017.09.004.

19. Lund, J.; Baumbach, J.; Li, S.; Svane, A.M.; Hjelmborg, J.; Christiansen, L.; Christensen, K.; Redmond, P.; Marioni, R.E.; Deary, I.J.; et al. DNA methylation linked to all-cause mortality in the elderly: An epigenome-wide association study. 2018, in progress. 1576-1584.

20. Deary, I.J.; Gow, A.J.; Pattie, A.; Starr, J.M. Cohort Profile: The Lothian Birth Cohorts of 1921 and 1936. Int. J. Epidemiol. 2012, 41, 1576-1584.

21. Finkel, D.; Pedersen, N.L. Processing Speed and Longitudinal Trajectories of Change for Cognitive Abilities: The Swedish Adoption/Twin Study of Aging. Aging Neuropsychol. Cognit. 2004, 11, 325-345.

22. Houseman, E.A.; Accomando, W.P.; Koestler, D.C.; Christensen, B.C.; Marsit, C.J.; Nelson, H.H.; Wiencke, J.K.; Kelsey, K.T. DNA methylation arrays as surrogate measures of cell mixture distribution. BMC Bioinform. 2012, 13, 86 .

23. Leek, J.T.; Johnson, W.E.; Parker, H.S.; Jaffe, A.E.; Storey, J.D. The sva package for removing batch effects and other unwanted variation in high-throughput experiments. Bioinformatics 2012, 28, 882-883.

24. Meinshausen, N.; Bühlmann, P. Stability selection. J. R. Stat. Soc. Ser. B 2010, 72, 417-473.

25. Mogensen, S.W.; Petersen, A.H.; Buchardt, A.S.; Hansen, N.R. Survival prognosis and variable selection: A case study for metastatic castrate resistant prostate cancer patients. F1000Research 2016, 5, 2680. 
26. Harrell, F.E.; Califf, R.M.; Pryor, D.B.; Lee, K.L.; Rosati, R.A. Evaluating the yield of medical tests. J. Am. Med. Assoc. 1982, 247, 2543-2546.

27. Harrell, F.E.; Lee, K.L.; Califf, R.M.; Pryor, D.B.; Lee, K.L.; Rosati, R.A. Regression modeling strategies for improved prognostic prediction. Stat. Med. 1984, 3, 143-152.

28. Larriba, S.; Sumoy, L.; Ramos, M.D.; Giménez, J.; Estivill, X.; Casals, T.; Nunes, V. ATB0/SLC1A5 gene. Fine localisation and exclusion of association with the intestinal phenotype of cystic fibrosis. Eur. J. Hum. Genet. 2001, 9, 860-866.

29. Bhutia, Y.D.; Babu, E.; Ramachandran, S.; Ganapathy, V. Amino Acid Transporters in Cancer and Their Relevance to 'Glutamine Addiction': Novel Targets for the Design of a New Class of Anticancer Drugs. Cancer Res. 2015, 75, 1782-1788.

30. Liu, Y.; Yang, L.; An, H.; Chang, Y.; Zhang, W.; Zhu, Y.; Xu, L.; Xu, J. High expression of Solute Carrier Family 1, member 5 (SLC1A5) is associated with poor prognosis in clear-cell renal cell carcinoma. Sci. Rep. 2015, 5, 16954.

31. Kaira, K.; Sunose, Y.; Arakawa, K.; Sunaga, N.; Shimizu, K.; Tominaga, H.; Oriuchi, N.; Nagamori, S.; Kanai, Y.; Oyama, T.; et al. Clinicopathological significance of ASC amino acid transporter-2 expression in pancreatic ductal carcinoma. Histopathology 2015, 66, 234-243.

32. Kriebel, J.; Herder, C.; Rathmann, W.; Wahl, S.; Kunze, S.; Molnos, S.; Volkova, N.; Schramm, K.; Carstensen-Kirberg, M.; Waldenberger, M.; et al. Association between DNA methylation in whole blood and measures of glucose metabolism: KORA F4 Study. PLOS ONE 2016, 11, e0152314.

33. Kinoshita, S.; Akira, S.; Kishimoto, T. A member of the C/EBP family, NF-IL6 $\beta$, forms a heterodimer and transcriptionally synergizes with NF-IL6. Proc. Natl. Acad. Sci. USA 1992, 89, 1473-1476.

34. Poli, V. The Role of C/EBP Isoforms in the Control of Inflammatory and Native Immunity Functions. J. Biol. Chem. 1998, 273, 29279-29282.

35. Pless, O.; Kowenz-Leutz, E.; Knoblich, M.; Lausen, J.; Beyermann, M.; Walsh, M.J.; Leutz, A. G9a-mediated Lysine Methylation Alters the Function of CCAAT/Enhancer-binding Protein- $\beta$. J. Biol. Chem. 2008, 283, 26357-26363.

36. Kim, T.W.; Kang, Y.K.; Park, Z.Y.; Kim, Y.H.; Hong, S.W.; Oh, S.J.; Sohn, H.A.; Yang, S.-J.; Jang, Y.J.; Lee, D.C.; et al. SH3RF2 functions as an oncogene by mediating PAK4 protein stability. Carcinogenesis 2014, 35, 624-634.

37. Tan, Q.; Heijmans, B.T.; Hjelmborg, J.V.B.H.; Soerensen, M.; Christensen, K.; Christiansen, L. Epigenetic drift in the aging genome: A ten-year follow-up in an elderly twin cohort. Int. J. Epidemiol. 2016, 45, 1146-1158

38. Hjelmborg, J.; Iachine, I.; Skytthe A.; Vaupel, J.W.; McGue, M.; Koskenvuo, M.; Kaprio, J.; Pedersen, N.L.; Christensen, K. Genetic influence on human lifespan and longevity. Hum. Genet. 2006, 119, 312-321. 